\title{
A Smartphone Intervention for Cycle Commuting
}

\author{
Yun-Maw Cheng \\ Institute of Design Science and Department of Computer \\ Science and Engineering \\ Tatung University \\ Taipei, Taiwan
}

\author{
Chao-Lung Lee \\ Department of Computer Science and Engineering \\ Tatung University \\ Taipei, Taiwan
}

\begin{abstract}
For those who new to cycling to and from work, how do you inspire yourself to keep up with it? Previous research has identified that having a partner is crucial to create and maintain a new habit. However, the rapidly changing work dynamics pose a challenge on this basis. Frequently failing to show up at the appointed or expected time can cause the motivation breakdown. In this paper, we introduce BikeTogether, a smartphone app that encourages and supports its users to cycle home with each other over the Internet. The app employs the metaphor of a bicycle flashlight to represent closeness, leading, and following between two sides. The cycling performance is also recorded so the users can track how they are doing over time. 10 participants were instructed and randomly paired to take a two-phases test ride on different routes. Results indicated that the app can help create the sense of being with each other while cycling and promote not only accompanied but competing ride. In addition, the outcome of the desirability towards the app implies a higher chance it will lead to a behavior change. This provides a new way that we can commit to remain encouraged.
\end{abstract}

Keywords-copresence; behavioral nudge; persuasive computing; smartphone app

\section{INTRODUCTION}

Fast paced lifestyle has compelled people to strike a balance between their work and health. Research has shown that exercising after work followed by getting a good night's rest is the essential to avoid physical and psychological exhaustion [1]. Cycling home after work can be a minimalistic approach that accomplishes the two tasks with only one effort [2]. Along with the benefit of the idea, people can simply make a positive change then repeat. However, this is easier said than done. The change of this kind is consequences of everyday decisions that assemble over time. Also, as the social comparison theory states: "Humans have a drive to assess how they are doing. In order to assess how they are doing they seek standards in which to compare themselves. When objective standards are not available, they look to their social environment and engage in comparison with available others" [3]. Social influences and supports play a critical role in encouraging and retaining the change [4]-[6].

Can the pervasiveness and technological capabilities of smartphones be designed to enable the change? As we have seen, they are not only for communication but also for measurement of variations in daily activities (i.e. status update, check-in, etc.). The use of smartphones together with various sensors is attracting attention of cycling communities. The information they provide is mainly about fitness and performance. The fitness information is about a cyclist's physical status, such as heart rate, pulse oximeter, skin temperature, galvanic skin response, or calories burned. The performance information is regarding a cyclist's cycling conditions, such as speed, time, and distance traveled. The information is then forged into the process of goal-setting, selfmonitoring, rewards and sharing features in order to better the cycling experience.

With all these, there are possibilities that can empower people to engage in collecting and sharing relevant real-time information to foster collective action based on their measurements [7][8]. To leverage the materialities of smartphones to embody the effect of social comparison on motivation for cycle commuting, there are questions to be answered [9][10]. What data are collected? How are the data turned into appropriate information and presentation, which is focused and meaningful enough for cycle commuters to understand and help them to make choices to hang on to the change?

These commuters get on their bicycles and let their legs carry them home. The exertions are transformed into acceleration and distance ridden. Mounting a smartphone on the handlebar to track these locomotion details and to share them between the two commuters in real-time is as handy as it is becoming. The sharing is considered a key in creating a sense of togetherness [11]. Also, let the information be contextual can support each of them to focus on their cycling without the necessity to reason it cognitively [12] [13]. The challenge herein is the selection of a suitable communication medium with its visibility and glanceability to carry the information in situ. Cycling home after work reminds us the importance of lighting. In addition to safety concerns, feeling isolated and alone is what augments the decreasing of the motivation. Lighting can create atmosphere and mood that makes us feel guided and accompanied [14][15]. Moreover, as Fogg suggests, flashing lights is one of the form factors that can motivate people and prompt immediate action [9]. Utilizing the screen of a smartphone as the lighting source to convey the exertion information in an ambient, unobtrusive, and intuitive manner could have the potential toward the goal of influencing the commuters to be more active.

Most people are already rely on the Internet as a source and a tool to broaden their circle of social interaction to include possibly everyone who share the same interests, beliefs, and goals. The mobility of smartphones can offer support for sensory extension to enable remote individuals to continue interacting with one another while moving around. Using 
dynamic patterns of light, such as color and intensity, on the screen of a smartphone as sensory cues to define the relationship between the cycle-commuters' perception and their cycling activity provides a tangible approach in creating a remote sense of togetherness. In response, we developed BikeTogether, an app to explore the possibility of using smartphones mounted on the handlebars as an ambient display to convey information that embodies social comparison to the commuters engaged in their cycling. To evaluate this experience, a series of studies were conducted to realize how those new to cycle-commute interpreted and responded to the design. In brief, this research makes the following contributions:

- It identifies that smartphone apps can be designed to create a sense of togetherness between two cyclecommuters while cycling. This leads to positive behavioral effects.

- It identifies what and which visualization and where to present information using a smartphone while cycling to embody remote presence.

- It confirms the feasibility of this approach through experiments on our prototype implementation via an inthe-wild study. The experimental results show that the app exhibits a good degree of persuasion.

\section{DESIGN GOALS AND CHOICES}

BikeTogether incorporates social comparison theory with the materiality of smartphones and ambient display. This section describes the design goals we want to achieve with our design choices. In order to know more about the motivation behind cycle-commuting, a brief interview of 5 colleagues in our department who cycle-commute at least on a part-time basis (1 female, 4 males, $\mathrm{M}=54.6$ years, $\mathrm{SD}=3.92$ ) was performed. The results showed that getting home quicker and feeling of healthier in terms of psychological, physical, and environmental outcomes hold prime importance in their choice of the commuting option. All of them expressed the feeling of wanting others to share in their enthusiasm. Also, a street interview of 15 random people ( 6 female, 9 males, $M=36.86$ years, $\mathrm{SD}=13.7$ ) waiting in line for a bicycle at a YouBike station, which is the public bicycle rental scheme in Taipei, during after work rush hour revealed reasons for cycling home other than what the colleagues expressed. Their responses included: it is cheaper because the YouBike provides free for the first 30 minutes promotion; I want to fit in the popular crowd with the idea of leaving the environment in a better condition. These reasons suggested that it has potential to encourage people to cycle-commute and keep up the change through the conversation with the like-minded others in situ.

There may be people with the same interests around can help keep us motivated. But we do not bump into each other as we expected to be. So go beyond our naked sense perceptions to reach each other in order to get motivated and inspired to do something that matters together is the challenge that the design of BikeTogether attempted to address. Those cycle-commuters are related by what they share in common but unknown to each other. The app is aimed at creating a form of conversation that enables its users to freely express to each other in the comprehension that they might never meet again [16][17]. This comprehension, however, intends to intrigue the thought of who and where the users really are to maintain the surprising freshness. Therefore, the level of anonymity the app adapts is that the users' locations are veiled while their identities are disclosed.

As mentioned, the app is designed to create a sense of togetherness using light as a communication medium regardless of the users' physical distance. The sense of being together with each other is a subjective experience. To fabricate this illusion, a synchronous communication between the two sides is needed. Also, finding intuitive and accessible manipulations in situ to reconfigure those preconceptions of smartphone use through the ambient engagement of the viewer and the use of visual sensory mapping strategies should be adopted. The strategies would then position the users as active producers of meanings to each other. This draws attention to the ways in which smartphones can bridge the two sides of users who want to do something together but cannot find each other in an implicit and unobtrusive manner. Along with this idea, there are also the questions of what the core messages in the conversation is and what the concept they mutually agree.

People have a radical desire to assess their ability [3]. It is, as a matter of course, the levels of exertion measured by acceleration and cycling distance are chosen as the core messages. Dubberly and Pangaro argued, "Conversation is a progression of exchanges among participants. Each participant is a 'learning system,' that is, a system that changes internally as a consequence of experience" [18]. The idea of BikeTogether is to provide its users with an informative and motivating representation of the core messages using light based on a self-reflective approach.

The light intensity is to be proportional to the acceleration during a cycling activity. In order to inform the users about how they are doing during their cycling, cumulative values of the distance ridden so far can be summed up, synchronously exchanged between the two sides of the users, and displayed in an ambient manner as the current ongoing overall result on the screen of the smartphone as colors. A green, for instance, could signify that the user has obtained a leading position compared to the other user, while a red would indicate that the user is on following position because the cycling distance is less than the user's on the other side. An orange indicates the closeness of two sides of the users.

The key thing about cycle-commuting is not simply to set a goal and to turn into a self-starter instantly. It has to be accomplished in a way that people feel they can now manage things they want to do better than before. BikeTogether aims to make it possible for the users to have a feeling of "I have a chance to meet a new partner every time when I cycle home". The feeling of togetherness, therefore, purposely encourages the users towards a desired behavior. Another deliberation for the users is to provide cumulative information about the total cycling distance since the app is taken on. In some way, it encourages the users to compete against themselves. This not only helps them reflect on cycle-commuting which is something they build up to but also the effect of feeling of togetherness on the rate of progress they perceive. The results 
may lead to develop from a personal need for achievement to an interactional and varying achievement goals. As to the design, the metaphor of a car mileage meter is used to describe the increase that occurs at each step of the progress. Over time, after glancing and contemplating the numeric difference, the sensation that we have a hope of accomplishment with likeminded others may grow stronger [19][20].

\section{INTERACTIONS OF THE BIKETOGETHER}

The goal of BikeTogether is to motivate people who just start cycle-commuting to do it more. In order to explore the design opportunities from the design choices discussed previously and to have a better understanding on how it carries out, the interactions are shown below:

1) The users simply grab their bicycles, mount their smartphones on the handlebars, and turn on the app. They then come to the selection screen, which is a flashlight with an on/off button as the only one major user interface element. The button is a circular menu with four features, "Double", "Solo", "History", and "Setting". The users can circle along the selection and press the on/off icon to confirm the selection.

2) When the app runs up for the first time, the user enters information such as identity and password, to create an account. This is all done by going to "Setting" in the circular menu.

3) When "Double" is selected, the outer edge of the button starts flashing in white and this indicates that the system is attempting to pair two sides of the users whoever are online. If it becomes solid white, this indicates that the connection is successfully established and the system starts to facilitate the exchange of distance data between the users since they are paired. Otherwise, the process is again repeated.

4) Also, the users can compete with their previous best times and efforts by selecting "Solo". The connection with your virtual self is made instantly once the button is pressed.

5) The flashlight illuminates in different colors. Red, green, and orange lights denote following, leading, and closeness between the paired users in terms of the distance the users have ridden. The intensity is directly proportional to the level of acceleration and the flickering means the current positional state is about to change.

6) When the on/off button is pressed second time by either side of the users, the pair is ended immediately and a review of the current results is then brought up. The user can check distance, average speed, time (duration and date), and paired partner's ID of the cycling. A route map is shown on the screen as well. The map displays the leading, closeness, and following distance, represented by green, orange, and red respectively.

7) The users can browse their cycling records by selecting "History" on the selection screen at any time. For a more elaborate look at any individual cycling activity, click the bar representing that cycling to review the route map and other details.

In the next section, an evaluation of the interaction and interface with users in the real scene is described. The hope is to discover missing elements or parts that can be smoother in encouraging people go for and keep up cycle-commuting.
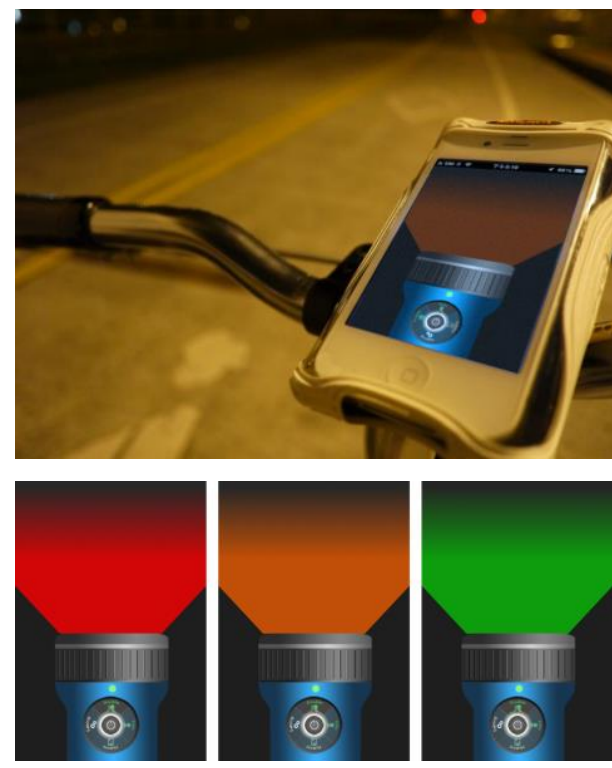

Fig. 1. BikeTogether in the field (top); Color changing of flashlight denotes the relative position, red: following, orange: closeness, green: leading (bottom).
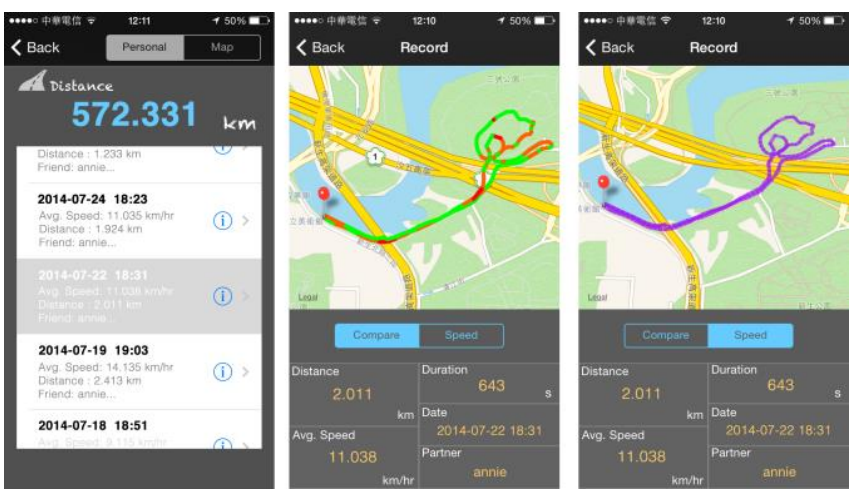

Fig. 2. Cycling history and performance.

\section{METHODOLOGY}

In order to understand if BikeTogether can effectively convey information to people engaged in a cycling activity via the screen light from a smartphone mounted on the handlebar, and whether this can influence their attitude towards the transit of this kind, a two-phase experiment was designed and carried out with real users in the Dajia Riverside bicycle path, which is one of the busiest paths for cycle-commuting in Taipei City. The participants consist of 10 undergraduate and postgraduate students at Tatung University (1 females, 9 males, $\mathrm{M}=23.1$ years, $\mathrm{SD}=1.14)$. The majority of the participants $(90 \%)$ use cycling as a recreation activity, and half of them prefer cycling with a companion. On the day prior to the scheduled experiment, participants received a notification via Facebook Messenger to remind and confirm the appointment. Also, they were all notified to wear comfortable clothing.

Two bicycles both with a smartphone mounted on the handlebars and BikeTogether preinstalled were prepared for 
the evaluation. The participants were then gathered, randomly split into 5 groups of two, and told to take a 10 minutes open cycle, which means they can ride on their own pace, on two different routes, one group at a time. As to how their cycling behavior is modeled, the mode of cycling is considered to be accelerating, decelerating, and constant speed in this evaluation. The cycling behavior of each participant therefore can be represented as an acceleration-time sequence within a certain time frame that meets the reality of cycling circumstances [21][22]. In this case, a time frame of 5 seconds was chosen.

These data were collected and calculated by the app ran in stealth mode without any GUI so the participants did not know they were being traced. After a 30 minutes rest, the participants were gathered once again and a brief instruction regarding the information coding of the app was given. They were then took another 10 minutes cycling with the app ran in normal mode, one group at a time. The data were again collected. Figure 3 shows the rate of acceleration and deceleration in both study phases. The specified threshold for count is $\pm 2 \mathrm{~m} / \mathrm{sec}^{2}$.

A Wilcoxon signed-rank test showed a statistically significant difference before and after participants were aware of BikeTogether $(\mathrm{W}=10, \mathrm{P} \leq 0.05)$. Also, to better understand how the app effected on the participants, their relative position to each other in terms of the real-time cycling distance in the second phase was summarized and an interview was performed.

\section{RESUlTS}

All the participants reported the increase in their activity level and considered that it was mainly due to the more aware of their personal activity as well as the comparison with remote partner's provided by the app. Group 4 said that it started like a subtle competition and later it became more like a group ride. Group 1 and 2 expressed that, at first, they attempted to attack off the front and later figure out the different physical ability in between, then slow down to their moderate pace. A common group cycling formation was seen in Group 3 and 5. They commented that once in leading position they would lower their speed in order for the other peer to catch up.

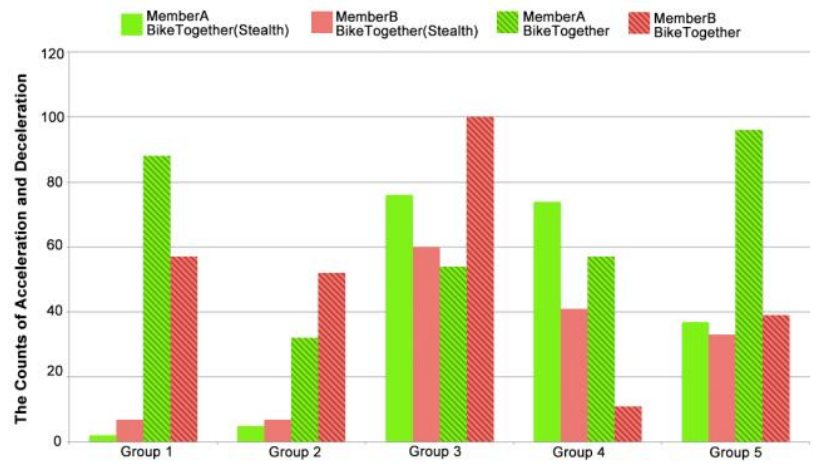

Fig. 3. The counts of acceleration and deceleration in each phase

In order to discover more about whether BikeTogether can engender positive effect that the users would use it frequently and longer, Production Reaction Cards was used to capture the participants' feelings and study their emotional involvement with BikeTogether [23]. These cards are composed of $60 \%$ of positive words and $40 \%$ of negative and neutral words. The participants were asked to select as many words as they wanted. The most selected word was fun $(72 \%)$, followed by entertaining (70\%), exciting (64\%) and motivating (51\%). All the participants also uttered that the total cycling distance directly provides them the feeling of achievement. This makes us have confidence in that BikeTogether can help further enhance the cycling experience and has potential to nudge people who just start cycle-commute to keep up the change.

\section{CONCLUSION AND FUTURE WORK}

Hard to find mutually convenient time and place with cycling partners is the common cause that hinders turning it into habit. The findings from this research indicate that smartphone apps can be designed to create a sense of togetherness between two cycle-commuters while cycling using the screen light. This is a form of instant messaging, a bicycle-to-bicycle, body-to-body conversation. It is even more like a form of networked, peer-to-peer augmented game. The one you chat and compete with, right at the moment, numbs your orientation in time. This leads to positive behavioral effects. The next stage will involve the usability tests and user experience evaluation of the prototype to see whether the increased feelings of presence increase motivation in the long term so that the health and fitness goals will be achieved?

\section{ACKNOWLEDGMENT}

This research is partially funded by the Ministry of Science and Technology grand 101-2221-E-036-010. Thanks to all our participants for taking part in this study and sharing your inspirations.

\section{REFERENCES}

[1] I. J. Nägel and S. Sonnentag, "Exercise and Sleep Predict Personal Resources in Employees' Daily Lives," Applied Psychology: Health and Well-Being, vol. 5, no. 3, pp. 348-368, October 2013.

[2] S. Handy, Y. Xing, and T. Buehler, "Factors associated with bicycle ownership and use: a study of six small U.S. cities," Transportation, vol 37, no. 6, pp. 967-985, Nov 2010.

[3] R. P. Larrick, K. A. Burson and J. B. Soll, "Social comparison and confidence: When thinking you're better than average predicts overconfidence (and when it does not)," Organizational Behavior and Human Decision Processes, vol. 102, pp. 76-94, December. 2006.

[4] A. S. Gabriel, J. M. Diefendorff, and R. J. Erickson, "The relations of daily task accomplishment satisfaction with changes in affect: A multilevel study in nurses," Journal of Applied Psychology, vol. 96, pp. 1095-1104, September 2011.

[5] M. Kanning and W. Schlicht, "Be active and become happy: An ecological momentary assessment of physical activity and mood," Journal of Sport and Exercise Psychology, vol. 32, pp. 253-261, April 2010

[6] E. Massung and C. Preist, "Normification: using crowdsourced technology to affect third-party change," In CHI '13 Extended Abstracts on Human Factors in Computing Systems (CHI EA '13), pp. 1149-1154, New York, ACM Press, 2013.

[7] S. Heggen, "Participatory sensing: repurposing a scientific tool for STEM education," interactions, vol. 20, no. 1, pp. 18-21, January 2013.

[8] J. van der Linden, Y. Rogers, and V. Waights, "A blended design approach for pervasive healthcare: Bringing together users, experts and technology," Health Informatics Journal, vol. 18, no. 3, pp. 212-218, September 2012.

[9] B. J. Fogg, D. Eckles, Mobile Persuasion: 20 Perspectives on the Future of Behavior Change; 1st ed.: Stanford Captology Media, 2007, pp. 7784. 
[10] T. R. Chang, E. Kaasinen, and K. Kaipainen, "What influences users' decisions to take apps into use?: a framework for evaluating persuasive and engaging design in mobile Apps for well-being," In Proceedings of the 11th International Conference on Mobile and Ubiquitous Multimedia (MUM '12), 2012, pp. 2:1-2:10.

[11] S. Zhao, "Toward a Taxonomy of Copresence," Presence: Teleoperators and Virtual Environments, vol. 12, no. 5, pp. 445-455, October 2003.

[12] V. Occhialini, H. van Essen, and B. Eggen, "Design and evaluation of an ambient display to support time management during meetings," In Proceedings of the 13th IFIP TC 13 international conference on Humancomputer interaction (INTERACT'11), ed. P. Campos, N. Nunes, N. Graham, J. Jorge, P. Palanque, pp. 263-280, vol 2, Berlin: SpringerVerlag, 2011.

[13] A. Göker, S. Watt, H. I. Myrhaug, N. Whitehead, M. Yakici, R. Bierig, S. K. Nuti, and H. Cumming, "An ambient, personalised, and contextsensitive information system for mobile users," In Proceedings of the 2nd European Union symposium on Ambient intelligence (EUSAI '04), pp. 19-24, New York, ACM Press, 2014.

[14] Y. Rogers, W. R. Hazlewood, P. Marshall, N. Dalton, and S. Hertrich, "Ambient influence: can twinkly lights lure and abstract representations trigger behavioral change?," In Proceedings of the 12th ACM international conference on Ubiquitous computing (Ubicomp '10), pp. 261-270, New York, ACM Press, 2010.

[15] C. Harrison, J. Horstman, G. Hsieh, and S. Hudson, "Unlocking the expressivity of point lights," In Proceedings of the SIGCHI Conference on Human Factors in Computing Systems (CHI '12), pp. 1683-1692, New York, ACM Press, 2012.
[16] H. C. Stuart, L. Dabbish, S. Kiesler, P. Kinnaird, and R. Kang, "Social transparency in networked information exchange: a theoretical framework," In Proceedings of the ACM 2012 conference on Computer Supported Cooperative Work, pp. 451-460, New York, ACM Press, 2012.

[17] R. Kang, S. Brown, and S. Kiesler, "Why do people seek anonymity on the internet?: informing policy and design," In Proceedings of the SIGCHI Conference on Human Factors in Computing Systems (CHI '13), pp. 2657-2666, New York, ACM Press, 2013.

[18] H. Dubberly and P. Pangaro, "On Modeling: What is conversation? How can we design for effective conversation?," interactions, vol. 16, no. 4, pp. 22-28, July 2009.

[19] J. McGonigal, Reality Is Broken: Why Games Make Us Better and How They Can Change the World, Penguin Books, 2011, part I.

[20] P. Lunenfeld, A. Burdick, J. Drucker, T. Presner and J. Schnapp, Digital_Humanities; the MIT Press, 2012, ch. 3-4.

[21] J. Dill and J. Gliebe, "Understanding and Measuring Bicycling Behavior: a Focus on Travel Time and Route Choice," Report, Oregon Transportation Research and Education Consortium (OTREC), 2008.

[22] K. J. Krizek, S. L. Handy, and A. Forsyth, "Explaining changes in walking and bicycling behavior: challenges for transportation research," Environment and Planning. B, Planning and Design, vol. 36, no. 4, pp. 725-740, January 2009.

[23] J. Benedek and T. Miner, "Product Reaction Cards," Microsoft, July 29, 2002 . 